\title{
A practical IP telephony application framework to conduct online surveys among University City students in China
}

\author{
J. Zhu \& E. Gide \\ Central Queensland University, Australia
}

\begin{abstract}
The purpose of this paper is to develop a practical online survey method by using IP telephony applications in order to understand the e-commerce consumer behaviour of "University City" students in China.

The researcher found that the beauty of using IP telephony is that the researcher may either see the students' gestures and facial expressions, or just listen by telling students both a conscious and unconscious effort to transfer much of the expression. It is beneficial for small businesses because of eliminating travel time and expense, reaching difficult-to-recruit participants, it is cost-effective, and increases production. In particular, personal questions can be addressed without geographical barriers.

Briefly, in this paper the following objectives have been achieved: firstly, the possibility to use IP telephony application to do the student survey online. Then, the benefits for small businesses to know China "University City" students online. Further, the practical way of designing and conducting virtual focus groups by the selecting and integrating samples to get the data more representative and precise have been researched. Finally, it has been identified that the most popular ICT tools in China are the Blogs and Chinese Messenger QQ.
\end{abstract}

Keywords: IP telephony, Nanjing "University City”, small business, online survey samples, Chinese ICP tool.

\section{Introduction}

This paper links IP telephony with online survey, in order to find a better way for small business to reach China "University City" students, in terms of their 
internet culture, campus internet culture, Internet behaviour. The author of the paper found the possibility of using IP telephony to conduct online survey and the benefits for small business by using this tool. Also, the author found two critical keys for researcher to successfully conduct online survey (1) soft skills (2) Chinese version software platform. Finally, a case study shows the whole process by using Chinese Messenger QQ, Blog, Campus BBS to do online survey in "Nanjing Jiangning University City".

\section{The possibility of using IP telephony to do an online survey}

\subsection{Definition of IP telephony}

Voice over Internet Protocol (also called VoIP, IP Telephony, Internet telephony, and Digital Phone, Web Telephony, WWW Telephony ) is the routing of voice conversations over the Internet or any other IP-based network. The voice data flows over a general-purpose packet-switched network, instead of traditional dedicated, circuit-switched voice transmission lines [2].

\subsection{Advantages}

\subsubsection{Cost}

In general, phone service via VoIP costs less than equivalent service from traditional sources but similar to alternative Public Switched Telephone Network (PSTN) service providers. Some cost savings are due to using a single network to carry voice and data, especially where users have existing under-utilized network capacity they can use for VoIP at no additional cost.

\subsubsection{Functionality}

VoIP can facilitate tasks that may be more difficulty to achieve using traditional phone networks: Incoming phone calls can be automatically routed to your VoIP phone, irrespective of where you are connected to the network. Take your VoIP phone of where you are connected to the network. Take your VoIP phone with you on a trip, and anywhere you connect it to the Internet, you can receive your incoming calls.

VoIP phones can integrate with other services available over the Internet, including video conversation, message or data file exchange in parallel with the conversation, audio conferencing, managing address books and passing information about whether others (e.g. friends or colleagues) are available online to interested parties.

\subsubsection{Mobility}

VoIP allows users to travel anywhere in the world and still make and receive phone calls:

- Subscribers of phone-line-replacement services can make and receive local phone calls regardless of their location. 
- Users of IM based VoIP services like Skype or Yahoo! IM can also travel anywhere in the world and make and receive phone calls.

\subsubsection{ADSL internet access}

VoIP requires broadband internet access. ADSL allows VoIP financially more appealing because it is combined with cable Internet access, which does not require a traditional phone line to operate

\subsection{Digital campus in China}

In China there are 100 million internet users, 30\% are university students. Students can access internet from different channels with ADSL [6]

- Campus computer lab : US\$ 0.30/hour

- Accommodation internet access: US\$0.50/month

- Internet café around the campus (24 hours, 7 days): US\$0.30/hour

- Home-based access for local students :US\$10.0/month

Lots of IP telephony software, like Yahoo messenger, MSN messenger, Skype can reach China. Also, China has its own Chinese edited IP telephony, for example, QQ is very popular among students.

As a result, it is possible and feasible to do online survey via IP telephony in China.

\section{The benefits for small businesses to reach China "University City" students online}

\subsection{It is marriages of online and phone methodologies}

It is easy to recruit students who have a second telephone line, or high speed access, and can be on both their computer and the phone at the same time. Usually, the information is posted for viewing on a website or sent out to participants and then discussed during the telephone group. This is one of the best marriages of the two methodologies. The connection and humanness and audio cues from the tfg and the visual data and use of the internet as a tool [4].

\subsection{Other benefits}

- Elimination travel time and expense

- Reaching difficult-to-recruit participants

- Cost effective

- Greater production

- Immediate transcripts

- Easier to moderate

- Easier to moderate

- $\quad$ Less swayed by group, less reticent to speak

- Personal question can be addressed

- Cross-country regardless the geographical barriers 
- $\quad$ Bulleting board style allows for participation on respondents schedule. This is a truly exciting breakthrough. It has sampling advantages, such as better mixture of people within the group, mixing heterogeneous and conflicting participants, mixing people to talk about sensitive topics and people who would be reluctant to participate together because they are competitors in a local geographical region; Online is not as dependent upon facility availability.

- More available in certain places than telephone conferencing facilities. Students can access local internet café, which is 24 hours, 7 days.

\section{The practical way of designing and conduction virtual focus groups and telephone groups}

\subsection{The ways of designing and conducting virtual focus groups and telephone groups}

The design and development activities necessary to conduct successful online groups are similar to offline groups, specifically: establishment of objectives, screeners, discussion guides, moderation, analysis and report writing.

- Objectives, screener, recruitment, and virtual facilities :

- Online groups: (real-time or asynchronous) can be initiated by contacting a moderator in online focus groups. Respondents can be recruited electronically from established panels, compiles online lists, targeted websites, or client-provided lists. Sometimes, telephone recruiting is utilized to recruit audiences.

- Discussion Guide Development and Design: the content and structure of the inquiry are mostly written in full sentence form, in advance. The main topic questions must be written clearly and completely

- Bulletin board groups: last 3 to 5 days or more depending on the amount of material to be covered.

- Show Rates and selection: Show rates can vary dramatically based on a number of factors, including: the target respondent; origination of the respondent (e.g. online database, established panel, website intercept, client customer or employee lists, etc.); confirmation procedures (e.g. online only, phone only, or phone and online combined); and the typical kinds of other personal/business commitments that can inhibit attendance. One positive influence is that the weather, traffic, and transportation have less of a negative impact on show rates

- Moderating: the method that is more frequently available and workable is having the discussion guide document loaded in a separate window behind the virtual room to use for cutting and pasting each question.

Online groups demand that a moderator possess strong and fast keyboard skills or be willing to hire an assistant who does. Also, moderating online groups requires someone who relates to the online venue and recognizes that respondents are adept at developing relationships in this medium. Many respondents have and/or do 
participate in chat rooms and feel comfortable relating online. At the same time, it is the responsibility of the moderator to help make the less experienced respondents feel comfortable and valuable. In addition, if topics not covered in the guide are introduced, the moderator can choose to interject a new line of questioning.

- View for the respondents

- Analysis and reporting [1].

\subsection{Online groups}

The two most utilized online methodologies are real-time, virtual focus group rooms where 6-8 respondents participate simultaneously and asynchronous online bulletin boards with (ideally) twelve to twenty respondents lasting over a period of days. Both venues allow respondents to participate from their desired location and, with the bulletin board, at a time that is most convenient for them. There are a variety of real-time and asynchronous virtual facilities offering different formats. The specific features, capabilities, and sophistication will vary according to which provider is selected. Online groups are limited in 6-8 people.

\subsection{Telephone groups}

There is tremendous richness of information in the human voice. When people are on the phone, their voices become even more expressive because they know subconsciously that they can't be seen.

\section{$5 \quad$ Requirements of expertise skills}

Conducting successful online groups requires the researcher adequate keyboard skills, or He/she may hire a keyboard assistant.

Telephone groups demand that a moderator possess hospitable tune and manner to communicate with the students in other side.

In addition, the researcher should get training to have the following soft skills:

- Listening skills: focus on the keys words, and extend certain questions if necessary

- Writing skills: in particular, for the bulletin. Outstanding topic can attract more students to click and browse what you post, more range to recruit students

- Presentation skills: the way the researcher talk should be simple, straight away, and impressive, but persuasive.

- Networking skills: network students leaders, professor, and students managers in advance, can be very supportive.

- Leadership skill: good leader can direct students on the track, ignoring the distractive things

- Negotiation skill: learn to get compromising with students in scheduling the time, in topics; be flexible and dynamic. 


\section{Case study}

\subsection{Nanjing Jiangning University City}

"Nanjing Jiangning University City" with 12 Universities is located inside Nanjing Jiangning Science Park. It is 50 kilometres away from main city "Nanjing". Currently 60,000 students study and live there. In three years, it expects to expand to 15 universities with 200,000 students filling in [10].

Students can access internet from different channels: campus computer lab, accommodation inside the campus, and internet café outside the campus which is open 24 hours, 7 days. All broadband internet access.

\subsection{Virtual focus groups and telephone groups}

\subsubsection{Objectives}

(1) University students internet culture (2) University students campus internet culture (3) "Networking" and "Searching" usefulness test (4) popular Chinese ICT tools (5) Role of University student on internet

\subsubsection{Advertising}

draft the introduction of researcher herself, and students she wants to meet;

\subsubsection{Bulletin board}

www.xici.net is a local website; it connects to Chinese version IP telephony QQ. The researcher registered on this website, setting up "particular discussion room" for each different University. Post survey Objectives, introduce the researcher herself with her life photos, and post advertising 24 hours in advance to recruit students. Then, in a couple of days, she got the response who wants to be Online Focus group (select 6 students for real time, 12 students for bulletin), who wants to be on Telephone Focus group (select 6 students)

\subsubsection{Sample selecting and integrating}

It is a special kind of purposive sample in selecting a group of geographical areas to represent a larger area. Its aims are to be more representative than a random sample including a wide range of extremes [5].

6.2.4.1 Stratification Using census data from small areas. (1) find census data about each campus of the whole "University City" area. (2) some way of selecting the sample within each campus [3].

6.2.4.2 Quota samples Use appropriate control variables:

- Gender

- $\quad$ Education level (diploma, bachelor, master, PhD)

- University

- Faculty/Discipline

- Income

- Knowledge/skills of computer use

- History in web-access

- Interests 
Table 1.

\begin{tabular}{|c|c|c|c|c|c|}
\hline \multirow{2}{*}{$\begin{array}{l}\text { Nanjing Jiangning } \\
\text { University City }\end{array}$} & \multirow{2}{*}{$\begin{array}{r}\text { Population } \\
(2006) \\
\text { '000s }\end{array}$} & \multirow{2}{*}{$\begin{array}{l}\% \text { of total } \\
\text { population }\end{array}$} & \multicolumn{2}{|c|}{ Clusters } & \multirow{2}{*}{$\begin{array}{r}\% \text { of } \\
\text { clusters }\end{array}$} \\
\hline & & & exact & rounded & \\
\hline & A & B & C & $\mathrm{D}$ & $E$ \\
\hline $\begin{array}{l}\text { Nanjing Medical } \\
\text { University }\end{array}$ & 10,000 & 16.6 & 3.98 & 4 & 16.6 \\
\hline $\begin{array}{l}\text { Nanjing Southeast } \\
\text { University }\end{array}$ & 12,000 & 20.0 & 4.8 & 5 & 20.8 \\
\hline $\begin{array}{l}\text { Nanjing Hohai } \\
\text { University }\end{array}$ & 9000 & 15.0 & 3.6 & 4 & 16.6 \\
\hline $\begin{array}{l}\text { Nanjing University of } \\
\text { Aeronautics and } \\
\text { Astronautics }\end{array}$ & 12,000 & 20.0 & 4.8 & 5 & 20.8 \\
\hline $\begin{array}{l}\text { Nanjing Industry } \\
\text { University }\end{array}$ & 8000 & 13.3 & 3.19 & 3 & 12.5 \\
\hline \multirow[t]{2}{*}{$\begin{array}{l}\text { Nanjing Xiaozhuang } \\
\text { Normal college }\end{array}$} & 9000 & 15.0 & 3.6 & 3 & 14.5 \\
\hline & 60,000 & 100 & 24 & 24 & 100.0 \\
\hline
\end{tabular}

6.2.4.3 Snowball samples In order to reach the diversified population in "University City", the feasible way to find its members is by asking others. First of all, find a few members (24) of the population ( focus group) - by any method (email, telephone, local website, Campus BBS, friends, etc.), this is the first round.

Then I asked each of these first-round members(students) whether they know of any students: flatmates, classmates, old friends. These names form the second round. Then, I go to each of those second-round people, ask them for more names.

6.2.4.4 Sample size If budget is limited, the rule-of-thumb is useful . Do two surveys: a small one first, to get some idea of the data ( 24 students), then a larger 
one. (200 students) with the experience I gained on the first survey, the second one is of higher quality.

6.2.4.5 e-Questionnaire design and publish Divide Questionnaire into four parts. In order to get the right information as precise as I can, I separated my Questionnaire into four parts: Website, Personal, Life style, Internet habit and use, which I can tell students Internet needs and e-commerce needs from different perspectives. Then, I used www.quetionpro.com [11] software to transfer Questionnaire into e-Questionnaire, then, translated into Chinese version, published on Chinese local website: www.xici.net .

\section{$7 \quad$ Tips for conducting virtual groups the first time}

- Moderator needs to thoroughly know Chinese version software platform and its capabilities.

- Estimate approximately two minutes a question, or 40-45 questions for a 90 minute group (leaves room for 10-15 follow-up probes during the group).

- Clearly define target segments and closely monitor screening and recruitment; rescreening can be conducted in the waiting room prior to the group.

- Educate observers and offer to demonstrate and explain technical aspects prior to the groups.

- Designate one client contact (Human Resource administrator better) to communicate with moderator.

- Complete discussion guide and all visuals one day before the groups and test to assure full functioning.

- Design discussion guide questions that are complete and concise and rewrite any question that might prompt a respondent to ask for clarification.

- Adopt Chinese version ICT tools, if the moderator doesn't speak Mandarin; always find a Chinese student to assistant him/her.

- Use local website to reach students, use local people to assistant the survey.

\section{Ways to avoid technical obstacles}

- Moderator should have excellent keyboard skills and expect multiple and simultaneous inputs from students and respondents.

- Moderator signs on at least 20-30 minutes prior to the group to establish connection, conduct last minute tests, observe waiting room, and confirm that the software is fully functioning.

- $\quad$ Schedule groups at least 30 minutes apart.

- Assign numbers to each discussion guide question and instruct respondents to use them in front of each of their answers. 
- Moderator submits questions in uppercase to facilitate easy recognition of moderator input during the group. This will also assist in reviewing the transcripts later.

- Check to make sure all browsers are compatible with Chinese version software platform.

- For any special tasks during the group (e.g., whiteboards, site visits) give thorough and clear directions before the task begins [1].

\section{Chinese ICT tools recommendation}

1. Chinese IP telephony recommendation: QQ mainly (90\% students), MSN messenger as supplement (50\% students) [8].

2. Chinese local website and reach campus BBS: access www.xici.net, where all Universities in Nanjing has Campus BBS. There are more than 20,000 register users. You may reach students as many as you can [7].

3. Ask students to connect you to their own Campus Intranet, where you can browse University policy, Students real life.

4. Ask students for their own Blog, and visit what they post, which you can understand their personal needs. Some students have already posted 200 articles, publishing their thinking, their opinions, their focus, and their specialised area.

5. Link to their own websites, and try to bridge their websites to other related websites.

\section{Conclusion}

The paper is about using IP telephony to reach China "University City" students to do online survey. With this survey method, budget, distance, and time are not barriers any more for small business to understand their target segments Chinese students. Chinese version IP telephony QQ, Blog [9], University Campus BBS and Chinese local software platforms are keys to meet students, recruit students, organize the focus groups, further involve students events and get insight of students real needs. Before the survey, the expertise should get the training properly in terms of soft skills to make sure they approach the students in the right way; also for those who can't speak Chinese, or whose boarding skill is not adequate, a student assistant is recommended to give a help; In addition, using a student administrator or a human resource staff in the research area to act the role of "contactor" can increase the reputation, and reduce the mistakes.

\section{References}

[1] Designing and Conducting Virtual Focus Groups www.sysurvey.com/tips/designing and conducting.htm

[2] Voice over IP http://en.wikipedia.org/wiki/Voip

[3] Sampling for surveys www.sysurvey.com/tips/sampling.htm 
50 The Internet Society II: Advances in Education, Commerce \& Governance

[4] Online Focus Group vs. Face to Face Focus Groups vs. Telephone Focus Groups http://www.mnav.com/online.htm

[5] Questionnaire Design and Surveys Sampling www.sysurvey.com/tips /arsham.htm

[6] CNNIC 2006, www.cnnic.net.com

[7] XICI, www.xici.net

[8] QQ, www.QQ.com

[9] SINA http://edu.sina.com.cn/y/g/blog/index.shtml

[10] NSP www.nsp.gov.cn/egnlish/coutactus.htm

[11] QUESTINPRO www.quetionpro.com 